\title{
Analysis of shearing deformation in rolling-spinning forming of Ti-6Al-2Zr-1Mo-1V tubes
}

\author{
Jing Guo, Mei Zhan ${ }^{\mathrm{a}}$, and He Yang \\ State Key Laboratory of Solidification Processing, School of Materials Science and Engineering, \\ Northwestern Polytechnical University, PO Box 542, Xi' an 710072, PR China
}

\begin{abstract}
In this work, an analytical study of the macroscopic deformation and microstructure evolution of Ti-6Al-2Zr-1Mo-1V alloys during rolling-spinning forming process was performed by developing Finite Element (FE) modeling using the ABAQUS/Explicit platform. The developed FE model was validated by comparing with experimental results. To investigate the shearing deformation during rolling-spinning process, representative nodes were selected in the FE model. The shearing strains of these nodes were obtained by modeling. The influence of shearing deformation in rollingspinning forming process on microstructures was also analyzed. An optimal selection of process parameters was obtained to achieve better microstructure.
\end{abstract}

\section{Introduction}

As key lightweight components for gas and liquid transport, long thin-walled tubes with good mechanical properties have been increasingly used in the aerospace, aviation and related hightechnology industries [1]. Nowadays, long thin-walled tubes are usually manufactured by flow spinning with advantages such as simple tooling, material saving, less loading [2, 3]. However, for some hardto-deform alloys such as titanium alloys, flow spinning still has its limitations of hard getting qualified tube blanks with large diameters. With this limitation, it is not easy to attain large diameter tubes with thinner wall by flow spinning for hard-to-deform alloys.

Ring rolling is an advanced incremental metal forming technology used to manufacture large dimension, precise seamless rings. With this technology, high performance rings with accurate geometry and good microstructure can be obtained $[4,5]$. Therefore, ring rolling process may be a good choice to manufacture qualified tube blanks used in flow spinning and this would overcome the limitation in flow spinning mentioned above. Combining the ring rolling and flow spinning process together, a new tube forming process called rolling-spinning can feed the need of manufacturing high qualified thin-walled tubes for hard-to-deform alloys.

During metal plastic forming process, besides tension and compression deformation, shearing deformation also plays an important role in deformation such as microstructure evolution [6-8].

\footnotetext{
${ }^{\text {a }}$ Corresponding author: zhanmei@nwpu.edu.cn
}

This is an Open Access article distributed under the terms of the Creative Commons Attribution License 4.0, which permits unrestricted use, distribution, and reproduction in any medium, provided the original work is properly cited. 


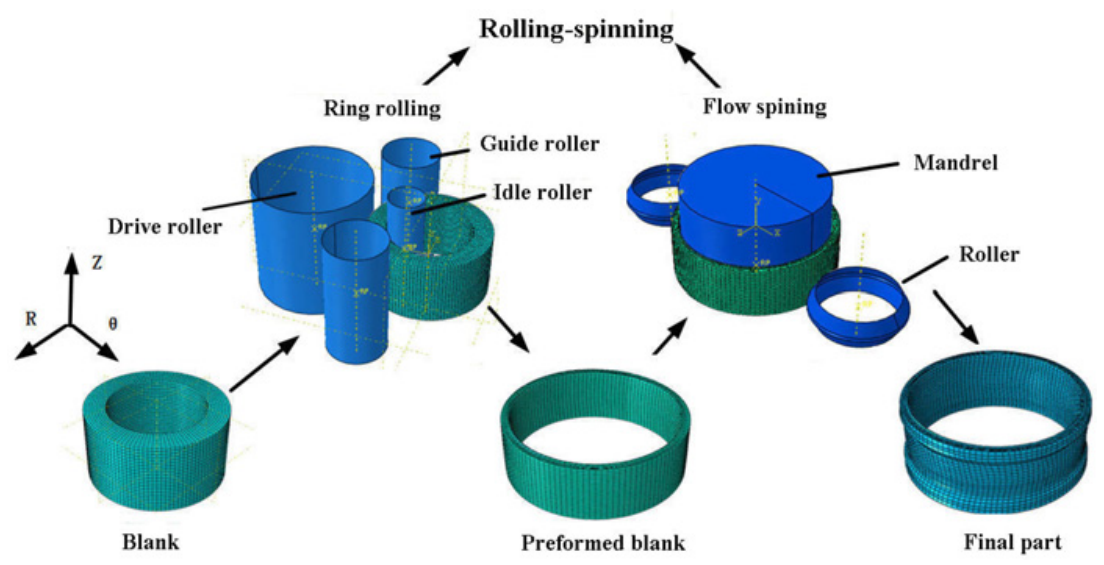

Figure 1. FE model of rolling-spinning process.

As a combination of two localized forming process, rolling-spinning forming process is a typical localized forming process. In this process, deformation is constrained strongly by surrounding metals and inhomogeneous metal flow occurs, which would result in strong shearing deformation in forming process. Zuo and Jiang used internal marks to investigate the shearing deformation in rolling process and concluded that the shearing deformation would make significant influences in strain and microstructure distributions. Besides this, the effects of process parameters on shearing deformation were also investigated [7]. Mohebbi and Akbarzadeh used Finite Element (FE) method to study the redundant strains in flow forming of tubes. Their results showed that the shearing deformation would bring redundant strains during flow forming process, and the magnitudes of shearing deformation would affect the dimension accuracy in flow forming of tubes [8]. These investigations in shearing deformation were only conducted in single forming process while for rolling-spinning process, which combines two different forming processes, shearing deformation would be different and needs to be further studied.

In this study, an FE model describing the macroscopic deformation and microstructure evolution of Ti-6Al-2Zr-1Mo-1V alloys in rolling-spinning forming process was established and then validated by comparing with the experimental results. Based on the FE model, the shearing deformation in rollingspinning process were investigated and analyzed.

\section{Establishment and validation of FE model}

Rolling-spinning forming process is a combination of ring rolling and flow spinning. Based on the previous FE models of ring rolling and flow spinning, a rolling-spinning FE model is established using ABAQUS/Explicit platform, as shown in Fig. $1[3,9,10]$. The main process parameters in rollingspinning process are listed in Tables 1 and 2. The cylindrical coordinate is also established in this model as shown in Fig. 1. In the rolling-spinning FE model, Continuous Analysis Technique is used to realize the modeling of whole rolling-spinning process. With this technique, the field outputs in ring rolling process can be transferred into flow spinning process and these transfers would make this model more close to actual experiments. The constitutive model of Ti-6Al-2Zr-1Mo-1V alloy used in the model is got from literature [3]. As rolling-spinning is a thermal and mechanical process, Temp-disp coupled module and C3D8RT meshes are used in ABAQUS/Explicit to calculate the rolling-spinning process. In addition, the dislocation density based internal-state-variables model is also embedded in rolling-spinning model by using user subroutine to redefine field variables (VUSDFLD) in ABAQUS to investigate the microstructure evolution in rolling-spinning process [11]. 
Table 1. Process parameters in ring rolling.

\begin{tabular}{|l|c|l|c|}
\hline Process parameters & Values & Process parameters & Values \\
\hline Radius of idle roller $(\mathrm{mm})$ & 30 & Blank thickness $(\mathrm{mm})$ & 30 \\
\hline Radius of drive roller $(\mathrm{mm})$ & 100 & Reduction $(\mathrm{mm})$ & 15 \\
\hline Radius of guide roller $(\mathrm{mm})$ & 50 & Feed speed $(\mathrm{mm} / \mathrm{s})$ & 1.5 \\
\hline Blank height $(\mathrm{mm})$ & 100 & Rotation speed $(\mathrm{rpm})$ & 60 \\
\hline Outer radius of blank $(\mathrm{mm})$ & 200 & Initial temperature $\left({ }^{\circ}\right)$ & 820 \\
\hline
\end{tabular}

Table 2. Process parameters in flow spinning.

\begin{tabular}{|l|c|l|c|}
\hline Process parameters & Values & Process parameters & Values \\
\hline Mandrel radius $(\mathrm{mm})$ & 154 & Feed speed $(\mathrm{mm} / \mathrm{s})$ & 3 \\
\hline Preformed blank height $(\mathrm{mm})$ & 100 & Rotation speed $(\mathrm{rpm})$ & 180 \\
\hline Outer radius of Preformed blank $(\mathrm{mm})$ & 169 & Reduction $(\mathrm{mm})$ & 5 \\
\hline Preformed blank thickness $(\mathrm{mm})$ & 15 & Roller radius $(\mathrm{mm})$ & 8 \\
\hline
\end{tabular}

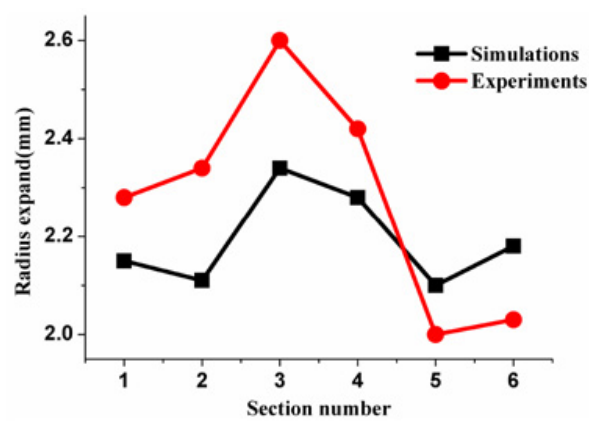

Figure 2. Comparisons of radius expand got in simulations and experiments.

Table 3. Comparison of grain size in experiments and simulation.

\begin{tabular}{|l|c|c|c|}
\hline Test number & Grain size (Experiments) $(\boldsymbol{\mu} \mathbf{m})$ & Grain size (Simulation) $(\boldsymbol{\mu} \mathbf{m})$ & Error \\
\hline 1 & 7.33 & 6.89 & $6.39 \%$ \\
\hline 2 & 5.23 & 5.05 & $3.56 \%$ \\
\hline
\end{tabular}

The comparisons between the values of radius expand got in flow spinning simulations for titanium alloys with experiments is shown in Fig. 2 [12]. The values of radius expand are got at different sections along axial directions. It can be found that the FE simulation results are in the same tendency at different sections and the maximum error is $11.5 \%$ compared with experiments, which indicates that the flow spinning model established in literature [12] is valid and reliable. As the rolling-spinning model in this study is established with the same methods in literature [12], the rolling-spinning FE model established in this study can be also thought to be reasonable and could be used to investigate the macro deformation behaviors in rolling-spinning. Besides this, the comparison of the grain sizes after various compression tests predicted by the VUSDFLD with the grain sizes got from compression experiments is also shown Table 3. The results indicate that the user subroutine could predict the microstructure evolution of Ti-6Al-2Zr-1Mo-1V in deformation accurately.

\section{Results and discussion}

\subsection{Shearing deformation in rolling-spinning}

The shearing deformation in rolling-spinning is investigated by representative nodes selected on the cross-section, longitude-section and outer surface of the FE model. The representative nodes on different 


\section{MATEC Web of Conferences}

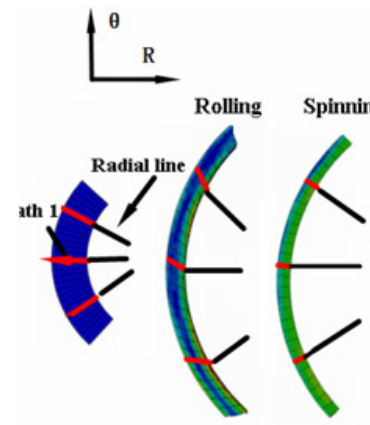

Cross-section

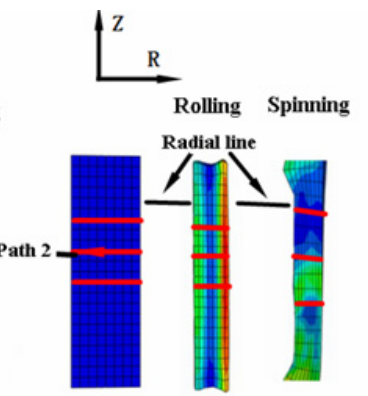

Longitude-section

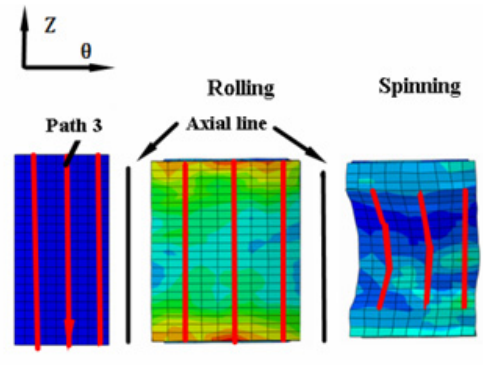

Outer surface

Figure 3. Inclinations of the lines on different sections after rolling-spinning.

sections are connected to lines, as shown in Fig. 3. Comparing the lines on different section with radial line or axial line in Fig. 3, the shearing deformation in rolling-spinning process can be observed directly. On the cross-section, the lines show obvious inclination compared with radial line after rolling, which indicates that shearing deformation occurs in cross-section during rolling process. While after spinning process, the lines on the cross section return to parallel to the radial line, which reveals that shearing deformation also occurs in cross-section in spinning process. On the longitude-section and outer surface, after ring rolling, comparing the lines with the radial line and axial line, it can be found that the inclinations of these lines are not obvious. While after spinning; the inclinations of these lines tend to be obvious. These phenomena indicate that shearing deformation in the longitude-section and outer surface mainly occurs during spinning process.

For the purpose to describe the shear deformation in rolling-spinning process quantitatively, engineering shear strains $\gamma_{R \theta}, \gamma_{Z R}$ and $\gamma_{Z \theta}$ are used and then got from the simulation results along particular paths in Fig. 3 and the node number is along the direction of arrow, as shown in Fig. 4(a). After rolling process, it can be found that $\gamma_{R \theta}$ are around 0.35 while $\gamma_{Z R}$ and $\gamma_{Z \theta}$ are around 0 . These indicate that shearing deformation occurs mainly in the cross-section in rolling process, which is also found in Fig. 3. The reason for these phenomena is that in ring rolling process, with the increase of radius and decrease of thickness, the materials in $R-\theta$ plane go through severe localized deformation which would cause inhomogeneous deformation. The inhomogeneous deformation would bring shear deformation $\left(\gamma_{R \theta}\right)$ in $R-\theta$ plane. Meanwhile, as blank height changes very little in rolling process, almost no shearing deformation $\left(\gamma_{Z R}, \gamma_{Z \theta}\right)$ appeared in $R-Z$ and $\theta-Z$ plane. Besides these, it is also found that $\gamma_{R \theta}$ are larger in both sides than the middle due to the direct effects of rollers. After spinning process, it can be seen that $\gamma_{R \theta}$ and $\gamma_{Z R}$ are about 0.25 , while $\gamma_{Z \theta}$ are about 0.1 . This is because that in spinning process, with the decrease of thickness and increase of length and radius, inhomogeneous deformation would occur and result in shearing deformation $\left(\gamma_{R \theta}, \gamma_{Z R}, \gamma_{Z \theta}\right)$ in all $R-\theta-Z$ planes. It can be also found that $\gamma_{R \theta}$ and $\gamma_{Z R}$ show an increase tendency along the path due to the direct effects of rollers. Besides these, $\gamma_{Z \theta}$ are found larger in both sides compared with those in the middle and this is caused by the roller effects on the upper side and tie constraint on the lower side. Based on the analysis above, it can be seen that in rolling-spinning process, large shearing deformation occurs through the cross-section while in single flow spinning process, shearing deformation mainly appear in the outer surface.

The distribution and magnitudes of equivalent plastic strain (PEEQ) and grain sizes along the crosssection in the rolling-spinning process are shown in Fig. 4(b). It can be seen that the distribution of PEEQ is similar with the distribution of shearing strain $\gamma_{R \theta}$ shown in Fig. 4(a), while small grain 


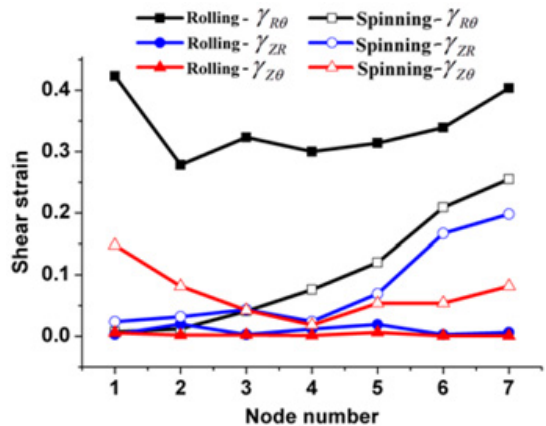

(a)

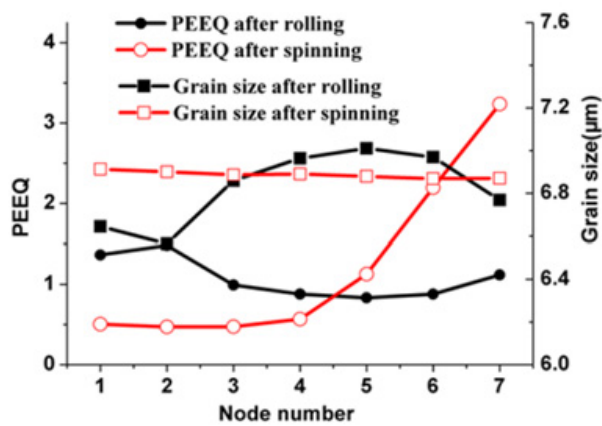

(b)

Figure 4. Shearing deformation in rolling-spinning process: (a) Distributions and magnitudes of shearing deformation in rolling-spinning (b) Distributions and magnitudes of PEEQ and grain size in rolling-spinning.

Table 4. Parameters conditions.

\begin{tabular}{|l|c|c|c|c|c|c|c|}
\hline Parameters & Ref & Sim1 & Sim2 & Sim3 & Sim4 & Sim5 & Sim6 \\
\hline Feed velocity $f_{s}(\mathrm{~mm} / \mathrm{s})($ rolling) & 1.5 & 3 & 5 & 1.5 & 1.5 & 1.5 & 1.5 \\
\hline Roller radius $r(\mathrm{~mm})(\mathrm{spinning})$ & 8 & 8 & 8 & 4 & 12 & 8 & 8 \\
\hline Feed velocity $f_{r}(\mathrm{~mm} / \mathrm{s})($ spinning) & 3 & 3 & 3 & 3 & 3 & 6 & 9 \\
\hline
\end{tabular}

sizes with uniform distribution are also found after rolling-spinning process. These illustrate that large shearing deformation would result in large PEEQ and small grains, which is also found in other literatures $[5,6]$.

\subsection{Parameters influences on shearing deformation in rolling-spinning}

As discussed in Sect. 3.1, large shearing deformation would result in small grain size. For the purpose to get tubes with small grain sizes by rolling-spinning process, the process parameters influences on shearing deformation are investigated by the single factor method. Roller feed velocity in rolling, roller radius and roller feed velocity in spinning are chosen as the parameters with three levels and the simulation scheme are listed in Table 4.

$\gamma_{Z \theta}$ and the grain sizes after rolling-spinning along path3 are used to describe the influences of $f_{s}$ on shearing deformation as shown in Fig. 5(a). It can be seen that $\gamma_{Z \theta}$ increases with the increase of $f_{s}$. This is because that large $f_{s}$ would lead to large deformation rate and large inhomogeneous deformation, thus bring large $\gamma_{Z \theta}$. In addition, it can be also found that the grain sizes are smaller and the distributions of grain sizes are more uniform under large $f_{s}$ than those small $f_{s}$. In Fig. 5(b), $\gamma_{R \theta}$ and grain sizes after rolling-spinning along path 1 are used to show the influence of $r$ on shearing deformation. Near the outer surface zones (nodes 5,6,7), $\gamma_{R \theta}$ are larger under the condition with $r=4 \mathrm{~mm}$ than those under bigger radius. This is because that the contact area for $r=4 \mathrm{~mm}$ is smaller than those larger radius and smaller contact area would bring larger $\gamma_{R \theta}$. Though $\gamma_{R \theta}$ are different under various roller radiuses, the grain sizes show no differences. To describe the influence of $f_{r}$ on shearing deformation, $\gamma_{R \theta}$ and grain sizes after rolling process along path1 are used, as shown in Fig. 5(c). Obviously, $\gamma_{R \theta}$ shows an increase tendency with the increase of $f_{r}$ in rolling as large $f_{r}$ would aggravate the inhomogeneous deformation in rolling. Besides this, it can be also seen that the grain sizes show the tendency of decrease with the increase of $f_{r}$. 


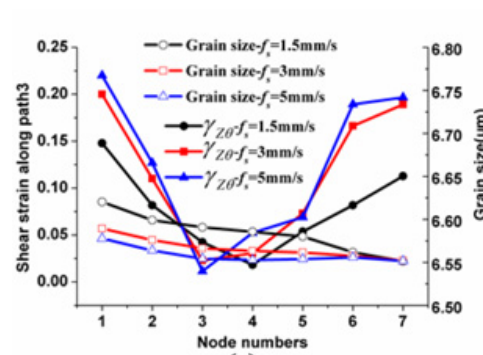

(a)

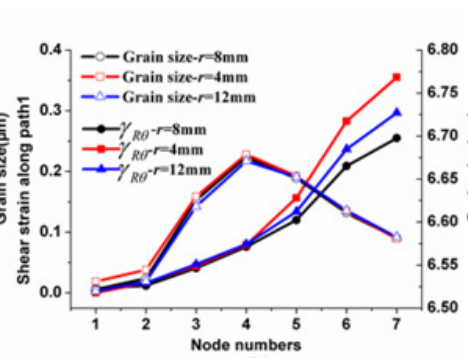

(b)

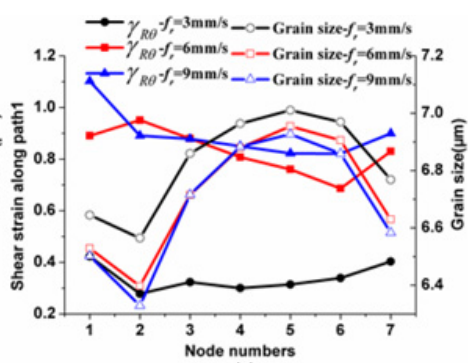

(c)

Figure 5. Shear deformation and grain sizes under different process parameters: (a) $f_{s}$ (b) $r$ (c) $f_{r}$

\section{Conclusion}

In rolling step before spinning process, the shearing deformation mainly occurs in the cross-section and the largest shearing deformation appears in the inner surface. While for the spinning step, shearing deformation occurs in the cross-section, longitude section and outer surface with that the largest shear deformation is in the outer surface. Based on the analysis of shear deformation in rolling-spinning, it can be found that large shearing deformation occurs through the cross-section and this would result in uniform distribution of small grain sizes. In addition, it is also found that larger feed velocity in rolling-spinning would bring larger shearing deformation and result in smaller grain sizes.

The authors would like to acknowledge the supports from National Science Fund for Excellent Young Scholars of China (Project 51222509); National Natural Science Foundation of China (Project 51175429); Research Fund of the State Key Laboratory of Solidification Processing (Projects 97-QZ-2014 and 90-QP-2013); and the EU Marie Curie Actions - MatProFuture Project (FP7-PEOPLE-2012-IRSES-31896).

\section{References}

[1] M. Zhan, T. Huang, H. Yang, J. Mater. Process. Technol. 217, 165 (2015)

[2] F.A. Hua, Y.S. Yang, Y.N. Zhang, M.H. Guo, D.Y. Guo, W.H. Tong, Z.Q. Hu, J. Mater. Process. Technol. 168(1), 68 (2005)

[3] D.B. Shan, G.P. Yang, W.C, X, J. Mater. Process. Technol, 209, 5713 (2009)

[4] X.H. Han, L. Hua, G.H. Zhou, B.H. Lu, X.K. Wang, Int J Mech Sci. 81, 95 (2014)

[5] D.S. Qian, Z.Q. Zhang, L. Hua, J. Mater. Process. Technol. 213, 1258 (2013).

[6] J. T. Wang, Z. Li, J. Wang, T.G. Langdon, Scripta Mater. 67, 810-813 (2012)

[7] F.Q. Zuo, J.H. Jiang, A.D. Shan, J.M. Fang, X.Y. Zhang, Trans. Nonferrous Met. Soc. China. 18, 774 (2008)

[8] M.S. Mohebbi, A. Akbarzadeh, J. Mater. Process. Technol. 210, 389 (2010)

[9] M. Wang, H. Yang, Z.C. Sun, L.G. Guo, X.Z. Ou, Trans. Nonferrous Met. Soc. China. 16, 1274 (2006)

[10] S. Zhu, H. Yang, L.G. Guo, R.J. Gu, Comp. Mater. Sci. 65, 221 (2012)

[11] X.G. Fan, H. Yang, Int J Plasticity. 27(11), 1833 (2011)

[12] D. Han, Doctor Degree Dissertation of Northwestern Polytechnical University (2013) (in Chinese) 